\title{
Docencia en Percepción Auditiva: adaptación a las necesidades de la realidad escolar
}

\author{
Esther Ruiz PaLomo \\ Universidad de Burgos (España) \\ erpalomo@ubu.es \\ Fernando LARA ORTEGA \\ Universidad de Burgos (España) \\ flara@ubu.es \\ Rosa M SANTAMARÍA CONDE \\ Universidad de Burgos (España) \\ rsantamaria@ubu.es
}

\section{Resumen:}

Las Universidades deben revisar periódicamente sus planes de estudio para responder a las demandas de la sociedad en la que se insertan y adecuar sus acciones formativas a las necesidades específicas del colectivo al que se dirigen.

Con esta premisa y tomando como referencia una serie de estudios realizados en nuestro país, se muestra en este trabajo un ejemplo de esta adaptación, aplicada concretamente al campo del Desarrollo de la percepción auditiva, partiendo de las necesidades formativas expresadas por el colectivo de maestros de educación infantil de la ciudad de Burgos.

Palabras clave: Formación; profesorado; percepción auditiva; educación infantil; educación musical; docencia universitaria.

\section{Teaching Hearing Perception: adapting to the needs of the school reality}

\begin{abstract}
:
Universities must periodically review their curricula to meet the requirements of the society in which they are acting and adapt its training to the specific needs of the target group.

With this premise and with reference to a series of studies conducted in our country, this paper is example of this adaptation, specifically applied to the area of development of hearing perception, based on the training needs expressed by the group of teachers in the elementary education in the city of Burgos.
\end{abstract}

Key words: Training; teaching; hearing perception; child education; music education; university teaching.

\section{Referencia normalizada:}

Ruiz Palomo, E., Lara Ortega F. y Santamaría Conde, R. M. (2014): Docencia en percepción auditiva: adaptación a las necesidades de la realidad escolar. Historia y Comunicación Social. Vol. 19. Núm. Especial Enero. Págs. 27-40

Sumario: 1. Introducción. 2. Metodología. 2.1. Objetivos. 2.2. Descripción del instrumento: El cuestionario. 2.3. Desarrollo de la investigación. 2.4. Valoración de los resultados. 3. Conclusiones y propuestas metodológicas. 4. Referencias bibliográficas. 4.1. Artículos en publicaciones web. 


\section{Introducción}

En palabras de López de la Calle (2008: 227): "Para formar un profesorado debidamente cualificado es necesario primeramente conocer cómo es la formación que los futuros maestros en educación infantil reciben en las universidades para utilizar la música en esta etapa y qué elementos pueden potenciar la adquisición de las capacidades básicas necesarias para utilizarla de manera segura y con garantías de calidad."

Los estudios realizados por Bernal (2000), Díaz (2004a) y López de la Calle (2009), han puesto de manifiesto la poca atención que se le presta a la educación musical en las aulas de infantil. En dichos estudios se parte, entre otras, de la hipótesis de que una de las causas que da lugar a esta circunstancia es que los futuros maestros de ese nivel educativo reciben una formación insuficiente en relación con la educación musical.

Coincidimos plenamente con López de la Calle (2008) cuando señala que se trata de una formación muy escasa, que no capacita para la implementación de la música en el currículum de la educación infantil y que no da respuestas a las nuevas exigencias musicales derivadas de las actuales estructuras educativas, culturales y sociales.

Díaz (2004b: 6) se pronuncia también en este sentido cuando dice:

Somos conscientes de que una mayoría del profesorado de Infantil demanda formación en educación musical. Por otra parte, la formación que están recibiendo los especialistas de Educación Infantil, que actualmente se forman en nuestras universidades, es a nuestro modo de entender muy escasa.

Tomando como referencia estos estudios y deseando conocer el estado de la cuestión en nuestra ciudad, llevamos a cabo, durante el curso 2011-2012, una investigación en los centros escolares que imparten $2^{\circ}$ ciclo de Educación Infantil en Burgos capital, avalada por la Dirección Provincial de Educación, con el objetivo de conocer qué formación inicial recibieron los maestros de Educación Infantil en sus centros formativos y recabar información sobre las carencias y necesidades que demandan en materia de Educación Musical.

Tras conocer los resultados de dicha investigación pusimos en marcha una serie de actuaciones que afectaban tanto a la formación inicial como a la formación permanente de los maestros.

Centrándonos en la formación inicial, que es el tema que nos ocupa en el presente trabajo, dichas actuaciones se concretaron en la revisión de la Guía Docente de la asignatura Desarrollo de la Expresión Musical y su Didáctica, seleccionando aquellos contenidos que, a raíz de las encuestas realizadas, eran más significativos para los maestros y aquellos en los que los encuestados solicitaban mayor formación, eliminando los que no eran imprescindibles, y en la puesta en práctica de una metodología docente que, sin olvidar la importancia de la teoría, incidiese en una formación más orientada a la práctica, mediante la aplicación inmediata a situaciones hipotéticas creadas en el aula y la realización de supuestos didácticos. 
Una vez realizados y puestos en práctica los cambios señalados procedimos a pasar a los alumnos el mismo cuestionario que habían respondido los maestros en ejercicio para comprobar la eficacia o no de las modificaciones introducidas en la docencia de la asignatura.

Mostramos aquí los resultados relativos a la formación recibida en materia de percepción auditiva y a la relevancia que para el colectivo tiene este contenido en su aplicación al aula ya que, como señalan distintos autores, la importancia de la educación musical en esta etapa viene dada por la necesidad de organizar en edades tempranas las percepciones auditivas de los niños para contribuir al desarrollo de su imaginación, sensibilidad y creatividad como fuente de sus posibilidades expresivas (Gallego Ortega, 1994).

Como señala Akoschky (1996), la escucha es uno de los contenidos fundamentales de la educación musical; todos los demás se irán hilvanando a través de la audición y la percepción. Es fundamental, en la educación musical infantil, favorecer el contacto de los niños con las experiencias sonoras que les ofrece su entorno más próximo, mediante un trabajo estructurado, con planteamientos y objetivos claros y a través de experiencias que irán desarrollando su sensibilidad y su capacidad de discriminación y de memoria auditiva.

El trabajo realizado debe encaminarse a desarrollar las facultades de concentración y atención y a distinguir entre oír y escuchar a través de juegos sonoros, siguiendo el proceso: Escuchar- Identificar- Retener- Reproducir (Ruiz, 2011).

El objetivo ha de ser despertar en los niños el interés auditivo, estimular su atención hacia cualquier fenómeno sonoro para sensibilizar su oído preparándolo para la comprensión y ejecución musical. El punto de partida será hacerles conscientes de su capacidad de percepción auditiva para que lleguen a descubrir, distinguir, ubicar, recordar, enumerar y seleccionar los sonidos que le rodean.

Por otra parte el desarrollo de la percepción auditiva tiene gran importancia no sólo desde un punto de vista musical, sino desde otros ámbitos. El trabajo auditivo fomenta la observación del entorno ya que la escucha orientada y dirigida del entorno social y natural proporcionará conocimientos que no permite el contacto permanente aunque indiscriminado con él (Akoschky, 1996); y este trabajo se hace más importante dado que vivimos en una sociedad cuyos estímulos son mayoritariamente visuales y no se presta adecuada atención a los procesos de escucha. Unido a esto, el desarrollo de la percepción auditiva también fomenta procesos de observación, manipulación, experimentación, etc., lo que contribuye al desarrollo cognitivo del niño.

Pero donde la percepción auditiva adquiere una especial importancia, además de en la educación musical, es en el ámbito de la comunicación y el lenguaje. La percepción auditiva constituye un prerrequisito para la comunicación. Implica la capacidad para reconocer, discriminar e interpretar estímulos auditivos asociándolos a experiencias previas. Es un elemento fundamental para el acceso y el desarrollo del lenguaje. Por vía auditiva nos llega cantidad de información de forma inconsciente e involunta- 
ria, pero, para entenderla, aprender a reaccionar y actuar sobre ella es imprescindible escuchar. Como señalan Calafi Rius et all. (2004: 9):

La percepción auditiva y un entrenamiento de las diferentes habilidades auditivas debe formar parte de las diversas actividades que los alumnos realizarán en el medio escolar $[\ldots]$.

La falta de comprensión y discriminación auditiva puede hacer que el niño no decodifique correctamente los elementos fónicos del lenguaje y no perciba diferenciaciones, produciendo errores en la imitación oral. El niño oye bien pero analiza e integra mal los sonidos que oye.

La capacidad auditiva se desarrolla en el niño en edades muy tempranas, por tanto, en el período educativo infantil se encuentra en su máximo desarrollo, por encima de sus capacidades motrices o vocales. Como venimos señalando, el desarrollo de la percepción auditiva es un contenido de gran importancia y, por lo que hemos podido observar de forma empírica, se trabaja muy poco en las aulas o se hace en forma poco adecuada.

El trabajo auditivo ha de ir encaminado a hacer nacer en el niño la conciencia de mundo sonoro, a lograr un desarrollo auditivo integral y consciente, a desarrollar su memoria auditiva y a estimular su capacidad de concentración auditiva mediante un trabajo progresivo y bien secuenciado. Se trata, en definitiva, de conseguir que el niño capte los sonidos y sus cualidades, reconozca la fuente que produce dichos sonidos, sea consciente de la amplia gama de sonidos naturales y artificiales existentes y pueda ubicarlos en el espacio.

Por la importancia señalada que adquiere el desarrollo de la percepción auditiva en diferentes ámbitos del desarrollo infantil consideramos fundamental conocer la formación que los maestros de este nivel educativo han recibido en relación con ella, así como la relevancia que para el colectivo tiene este contenido en sus prácticas diarias en el aula, con el fin de poder revisar nuestra docencia en la formación inicial y comprobar si las modificaciones efectuadas tras dicha revisión cumplen las expectativas y se convierten en un aprendizaje significativo que permita la integración del trabajo auditivo en las aulas de Educación Infantil de una forma estructurada, con objetivos claros y actividades adecuadas a cada una de las edades que comprenden dicha etapa.

\section{Metodología}

El diseño de ambas investigaciones presenta un carácter predominantemente descriptivo. La recogida de datos se he realizado a través de encuestas utilizando como instrumento el cuestionario, empleando, con algunas modificaciones, el cuestionario elaborado por López de la Calle Sampedro (2009). Son investigaciones tanto 
cualitativas, que permiten la interpretación de los datos, como cuantitativas, puesto que se han aplicado técnicas cuantitativas del programa SPSS.

Las variables analizadas son variables categóricas por lo que, en el análisis estadístico, hemos empleado tablas de contingencia acompañadas de las pruebas de Chi cuadrado que nos indicarán si las diferencias entre los grupos son significativas (valor $\mathrm{p}<0,05$ ) o no significativas (valor $\mathrm{p}>0,05$ ).

Los resultados que presentamos en este trabajo son aquellos alcanzados tanto por alumnos como por maestros en ejercicio, referidos a la formación recibida en relación con la percepción auditiva, al ser éste, y como ya hemos comentado con anterioridad, un contenido fundamental en las primeras edades del niño que, desde nuestro punto de vista, no se trabaja lo suficiente. Dichos resultados muestran diferencias muy significativas en cuatro de los seis ítems objeto de estudio.

\subsection{Objetivos}

La primera fase de la investigación tuvo como objetivos:

- Conocer qué formación inicial sobre Música y su Didáctica recibieron los maestros de Educación Infantil en sus centros formativos.

- Conocer qué formación perciben como más adecuada así como las carencias y necesidades del colectivo en materia de Educación Musical.

Los objetivos de la segunda fase se centraron en:

- Revisar y modificar nuestra práctica docente acercándola y adecuándola lo más posible a las necesidades expresadas por los encargados de llevar a cabo la tarea de trabajar la música en las aulas de Educación Infantil, esto es, los futuros maestros de la etapa.

- Comprobar mediante la aplicación de un cuestionario si las revisiones y modificaciones realizadas habían resultado eficaces.

- Diseñar y llevar a cabo acciones formativas fundamentadas en las necesidades y carencias expresadas por el colectivo encuestado así como promover trabajos de colaboración entre la Universidad de Burgos y los maestros dado que, una de las tareas de la Universidad ha de ser la de responder a las demandas que plantea la sociedad en la que se encuentra inmersa.

\subsection{Descripción del instrumento: el cuestionario}

Se ha empleado un cuestionario que consta de cuatro secciones muy concretas. La primera de ellas, de carácter más personal, recoge información acerca de los datos particulares de la persona encuestada, datos sobre su labor docente, Centro y año de finalización de sus estudios de Magisterio, si poseen o no formación y experiencias musicales ajenas a la formación recibida en la Universidad y si cuentan con la cola- 
boración del especialista en Educación Musical en la realización del trabajo musical en el aula.

En la segunda sección el encuestado debe reflejar su percepción en cuanto a la formación inicial recibida en materia de Educación Musical y el grado de conocimientos adquiridos. También se recoge la valoración que hacen de otros aspectos relevantes en la formación musical como: grado de formación del profesorado universitario, metodología empleada, tiempo dedicado a la teoría y a la práctica, etc.

En relación con la variable que nos ocupa en este trabajo los ítems sobre los que en esta sección se pregunta a los encuestados se recogen en la tabla 1:

\begin{tabular}{|l|l|l|l|l|}
\hline Formación para la comprensión musical: contenidos & Mucho & Bastante & Poco & Nada \\
\hline Conocimientos sobre el sonido y sus cualidades & & & & \\
\hline Conocimientos sobre el entorno sonoro & & & & \\
\hline Conocimientos sobre educación auditiva & & & & \\
\hline
\end{tabular}

Tabla 1. Fuente: Elaboración propia

La tercera sección se divide en dos apartados el primero de los cuales reúne información sobre la formación recibida en relación con los procedimientos musicales.

En la tabla 2 podemos ver los ítems relacionados con la percepción auditiva sobre los que se pregunta:

\begin{tabular}{|l|l|l|l|l|}
\hline Formación en procedimientos musicales & Mucho & Bastante & Poco & Nada \\
\hline Audición de sonidos y obras musicales & & & & \\
\hline
\end{tabular}

Tabla 2. Fuente: Elaboración propia

En el segundo apartado de ésta tercera sección se pregunta acerca de aquellos contenidos procedimentales que consideran más relevantes en su trabajo diario en relación con la música, en aspectos perceptivos, expresivos, de representación y de creatividad.

Los ítems acerca de los cuales debían responder (Tabla 3), son:

\begin{tabular}{|l|l|l|l|l|}
\hline \multicolumn{5}{|c|}{ Relevancia de los contenidos procedimentales básicos } \\
\hline PERCEPCIÓN & Mucho & Bastante & Poco & Nada \\
\hline Discriminación del entorno sonoro & & & & \\
\hline Discriminación de las cualidades del sonido & & & & \\
\hline
\end{tabular}

Tabla 3. Fuente: Elaboración propia

Finalmente la cuarta sección recoge las necesidades expresadas y el interés mostrado en cuanto a formación en materia de educación musical se refiere.

El total de ítems de las cuatro secciones permite realizar un estudio bastante pormenorizado de la formación musical de los docentes. 


\subsection{Desarrollo de la investigación}

El trabajo de investigación se ha llevado a cabo en dos fases, divididas a su vez en dos etapas.

En la primera fase la primera etapa se dedicó a la elaboración del cuestionario y su envío a los centros escolares facilitando a cada uno de ellos un dossier para el equipo docente de Educación Infantil, que contenía: tantos cuestionarios como maestros/as de infantil hubiera en el centro, una carta explicando los objetivos de la investigación y solicitando la colaboración del colectivo, así como un sobre timbrado con la dirección a la que se debían remitir los cuestionarios una vez cumplimentados. En principio fueron enviados 9 cuestionarios piloto, que sirvieron para revisar y corregir aspectos que no quedaban totalmente claros, tras lo cual se procedió a remitir el resto de cuestionarios.

La segunda etapa se dedicó a la recogida de los cuestionarios, clasificación y análisis de datos, elaboración de conclusiones derivadas de dicho análisis, principales aportaciones del estudio, presentación de resultados, etc.

En esta primera fase de la investigación, referida a la formación en educación musical recibida por los maestros de Educación Infantil, la muestra la compusieron todos aquellos docentes que imparten docencia en el $2^{\circ}$ ciclo de Educación Infantil de los centros escolares de Burgos capital. Se pasaron 236 cuestionarios, que fueron entregados en mano en visitas a cada uno de los 40 centros que reúnen las características expresadas. Respondieron 140 maestros de 32 centros, lo que supone un $60,5 \%$ de sujetos y un $80 \%$ de los centros escolares. Con éste porcentaje de respuestas consideramos que la muestra sí es representativa para nuestro estudio.

La segunda fase comenzó con la revisión de la Guía Docente de la asignatura Desarrollo de la Expresión Musical y su Didáctica y de la Metodología utilizada en la puesta en práctica de dicha materia con el fin de adaptarlas a las necesidades expresadas por los maestros en los cuestionarios, tras lo cual se procedió a:

- aplicar las revisiones realizadas tanto en relación con los contenidos como con la metodología docente, y

- preguntar a los alumnos, empleando el mismo cuestionario aplicado en la investigación precedente, acerca de la formación recibida, analizar los resultados y extraer conclusiones.

En cuanto a esta segunda fase, referida a la formación recibida por los alumnos del Grado de Maestro en Educación Infantil en cuanto a Educación Musical se refiere, la muestra la constituyeron los 144 alumnos matriculados en la asignatura. Recibimos 103 cuestionarios cumplimentados, es decir, un $71,52 \%$ de alumnos, lo que hace que la muestra sea suficientemente representativa para el estudio. 


\subsection{Valoración de los resultados}

Las variables, así como los diferentes ítems que componen cada una de ellas, relacionadas con la Percepción Auditiva y cuyos resultados presentamos en este trabajo, se pueden ver en las Tablas 1,2 y 3

En el análisis estadístico de los datos, y de cara a lograr una mayor claridad, hemos agrupado las categorías de Poco-Nada y Bastante-Mucho.

Reflejamos a continuación los resultados obtenidos en términos porcentuales.

En la formación para la comprensión musical (contenidos) se valoraban, como hemos visto, tres ítems: Conocimientos sobre el sonido y sus cualidades, Conocimientos sobre el entorno sonoro y Conocimientos sobre educación auditiva. Del análisis de los resultados de cada uno de ellos se derivan los siguientes porcentajes:

Conocimiento sobre el sonido y sus cualidades * Alumno o Maestro

\begin{tabular}{|l|l|l|r|r|r|}
\hline \multicolumn{2}{|c|}{} & \multicolumn{2}{|c|}{ Alumno o Maestro } & \multirow{2}{*}{ Total } \\
\cline { 4 - 5 } $\begin{array}{l}\text { Conocimiento sobre el } \\
\text { sonido y sus cualidades }\end{array}$ & Nada o poco & Recuento & 19 & 93 & 112 \\
\cline { 3 - 5 } & & Porcentaje & $18,4 \%$ & $66,9 \%$ & $46,3 \%$ \\
\cline { 3 - 6 } & Bastante o Mucho & Recuento & 84 & 46 & 130 \\
\cline { 3 - 5 } & & $81,6 \%$ & $33,1 \%$ & $53,7 \%$ \\
\hline
\end{tabular}

Tabla 4. Chi cuadrado $=55,882 ; \mathrm{p}<0,05$ (Estadístico exacto de Fisher: Sig. exacta, bilateral) . Fuente: Elaboración propia

Como se ve en la Tabla 4, el porcentaje de alumnos que responden que han recibido poco/nada formación es muy reducido en relación al de maestros, y viceversa, el de alumnos que responden que han recibido bastante/mucha formación supera en un $48,5 \%$ al de maestros en ejercicio. Se trata por tanto, y tal y como indica el valor de "p", de una diferencia muy significativa.

\section{Conocimientos sobre el entorno sonoro * Alumno o Maestro}

\begin{tabular}{|c|c|c|c|c|c|}
\hline & \multicolumn{2}{|c|}{ Alumno o Maestro } & \multirow[b]{2}{*}{ Total } \\
\hline & & & Alumno & Maestro & \\
\hline \multirow{4}{*}{$\begin{array}{l}\text { Conocimientos sobre } \\
\text { el entorno sonoro }\end{array}$} & \multirow[t]{2}{*}{ Nada o poco } & Recuento & 38 & 104 & 142 \\
\hline & & Porcentaje & $36,9 \%$ & $76,5 \%$ & $59,4 \%$ \\
\hline & \multirow{2}{*}{$\begin{array}{l}\text { Bastante o } \\
\text { Mucho }\end{array}$} & Recuento & 65 & 32 & 97 \\
\hline & & Porcentaje & $63,1 \%$ & $23,5 \%$ & $40,6 \%$ \\
\hline
\end{tabular}

Tabla 5. Chi cuadrado $=38,072 ; p<0,05$ (Estadistico exacto de Fisher: Sig. exacta, bilateral). Fuente: Elaboración propia

La tabla 5 muestra que, al igual que ocurría con el ítem anterior, también el porcentaje de alumnos que dicen haber recibido bastante/ mucha formación en Conoci- 
mientos sobre el entorno sonoro $(63,1 \%)$ supera al de maestros $(23,5 \%)$, y, aunque la diferencia no es tan grande $(39,6 \%)$ como la que se apreciaba en Conocimiento sobre el sonido y sus cualidades, se trata igualmente, como muestra el hecho de que "p" < 0,05 , de un valor significativo.

\section{Conocimientos sobre educación auditiva * Alumno o Maestro}

\begin{tabular}{|c|c|c|c|c|c|}
\hline & \multicolumn{2}{|c|}{ Alumno o Maestro } & \multirow[b]{2}{*}{ Total } \\
\hline & & & Alumno & Maestro & \\
\hline \multirow{4}{*}{$\begin{array}{l}\text { Conocimientos sobre } \\
\text { educación auditiva }\end{array}$} & \multirow[t]{2}{*}{ Nada o poco } & Recuento & 12 & 106 & 118 \\
\hline & & Porcentaje & $11,7 \%$ & $77,4 \%$ & $49,2 \%$ \\
\hline & \multirow{2}{*}{$\begin{array}{l}\text { Bastante o } \\
\text { Mucho }\end{array}$} & Recuento & 91 & 31 & 122 \\
\hline & & Porcentaje & $88,3 \%$ & $22,6 \%$ & $50,8 \%$ \\
\hline
\end{tabular}

Tabla 6. Chi cuadrado =101, 612; $p<0,05$ (Estadistico exacto de Fisher: Sig. exacta, bilateral). Fuente: Elaboración propia

En este caso (Tabla 6) la diferencia entre los maestros que dicen haber recibido bastante/mucha formación en este campo $(22,6 \%)$ y los alumnos $(88,3 \%)$ se hace notablemente mayor alcanzando el $65,7 \%$. Al igual que ocurría en los ítems anteriores, el valor $\mathrm{p}<0,05$ nos indica una diferencia muy significativa.

Por otro lado, en lo que se refiere a la formación en procedimientos musicales, con un único ítem: Audición de sonidos y obras musicales, destacamos los siguientes resultados:

Audición de sonidos y obras musicales * Alumno o Maestro

\begin{tabular}{|c|c|c|c|c|c|}
\hline & \multicolumn{2}{|c|}{ Alumno o Maestro } & \multirow[b]{2}{*}{ Total } \\
\hline & & & Alumno & Maestro & \\
\hline \multirow{4}{*}{$\begin{array}{l}\text { Audición de sonidos y } \\
\text { obras musicales }\end{array}$} & \multirow[t]{2}{*}{ Nada o poco } & Recuento & 34 & 111 & 145 \\
\hline & & Porcentaje & $33,0 \%$ & $79,9 \%$ & $59,9 \%$ \\
\hline & \multirow[t]{2}{*}{ Bastante o Mucho } & Recuento & 69 & 28 & 97 \\
\hline & & Porcentaje & $67,0 \%$ & $20,1 \%$ & $40,1 \%$ \\
\hline
\end{tabular}

Tabla 7. Chi cuadrado $=54,061 ; \mathrm{p}<0,05$ (Estadístico exacto de Fisher: Sig. exacta, bilateral). Fuente: Elaboración propia

Como ocurría en los 3 ítems de la variable anterior (tablas 4, 5 y 6) también en éste encontramos una diferencia significativa $(46,9 \%, \mathrm{p}<0,05)$ entre el porcentaje de alumnos $(67,0 \%)$ y el de maestros $(20,1 \%)$ que dicen haber recibido bastante/mucha formación en audición de sonidos y obras musicales, lo cual no deja de ser un dato revelador (Tabla 7).

Nos centramos por último en la variable referida a relevancia de los contenidos procedimentales básicos (percepción), y cuyos resultados en los dos ítems que componen la variable: Discriminación del entorno sonoro y Discriminación de las cualidades del sonido, son muy similares en ambas investigaciones: 
Discriminación del entorno sonoro * Alumno o Maestro

\begin{tabular}{|lll|r|r|r|}
\hline & & \multicolumn{2}{|c|}{ Alumno o Maestro } & \multicolumn{1}{c|}{} \\
\cline { 3 - 5 } & & \multicolumn{1}{c|}{ Alumno } & \multicolumn{1}{c|}{ Maestro } & \multicolumn{1}{c|}{ Total } \\
\hline \multirow{2}{*}{$\begin{array}{l}\text { Discriminación del } \\
\text { entorno sonoro }\end{array}$} & Nada o poco & Recuento & 17 & 28 & 45 \\
\cline { 2 - 5 } & $\begin{array}{l}\text { Bastante o } \\
\text { Mucho }\end{array}$ & Recuento & $16,5 \%$ & $21,2 \%$ & $19,1 \%$ \\
\cline { 2 - 5 } & Porcentaje & 86 & 104 & 190 \\
\hline
\end{tabular}

Tabla 8. Chi cuadrado =0,828; $p>0,05$ (Estadístico exacto de Fisher: Sig. exacta, bilateral). Fuente: Elaboración propia

En este caso, el porcentaje de alumnos que concede a la discriminación del entorno sonoro de bastante/mucha relevancia es de un $83,5 \%$ y el de maestros de un $78,8 \%$, con lo que la diferencia $(4,7 \% \mathrm{p}>0,05)$ no es significativa (Tabla 8$)$.

\section{Discriminación de las cualidades del sonido * Alumno o Maestro}

\begin{tabular}{|lll|r|r|r|}
\hline & & & \multicolumn{2}{c|}{ Alumno o Maestro } & \\
\cline { 3 - 5 } & & \multicolumn{1}{c|}{ Alumno } & \multicolumn{1}{c|}{ Maestro } & \multicolumn{1}{c|}{ Total } \\
\hline $\begin{array}{l}\text { Discriminación de } \\
\text { las cualidades del } \\
\text { sonido }\end{array}$ & Nada o poco & Recuento & 21 & 32 & 53 \\
\cline { 2 - 5 } & $\begin{array}{l}\text { Bastante o } \\
\text { Mucho }\end{array}$ & Recuenta & $20,4 \%$ & $23,9 \%$ & $22,4 \%$ \\
\cline { 2 - 5 } & Porcentaje & $79,6 \%$ & $76,1 \%$ & $77,6 \%$ \\
\hline
\end{tabular}

Tabla 9. Chi cuadrado =0,409; $p>0,05$ (Estadístico exacto de Fisher: Sig. exacta, bilateral). Fuente: Elaboración propia

Un hecho similar al anterior se produce en este segundo ítem (Tabla 9), en el que la diferencia entre los alumnos que la consideran bastante/muy relevante $(79,6 \%)$ y la de los maestros $(76,1 \%)$ se reduce a un $3,5 \%$.

El valor de $\mathrm{p}>0,05$ nos indica que se trata de una diferencia no significativa.

\section{Conclusiones y propuestas metodológicas}

Como se ha podido apreciar en los resultados, las diferencias entre la percepción que sobre su formación en materia de Educación Musical, concretamente en relación con la percepción auditiva, existen entre los maestros en ejercicio y los alumnos del Grado en Maestro de Educación Infantil son muy notables, tanto en los ítems que hacen referencia a la formación en contenidos como en aquellos que se refieren a la formación en procedimientos.

Estas diferencias se reducen considerablemente cuando a ambos colectivos se les pregunta acerca de la relevancia que le conceden a este contenido en concreto en su aplicación en las aulas infantiles. Es decir, independientemente de la formación reci- 
bida en cuanto a percepción auditiva se refiere, una gran mayoría considera bastante o muy relevante trabajar estos contenidos en el aula. El tiempo dirá si las revisiones realizadas a nivel tanto conceptual como metodológico han capacitado a los futuros maestros para implementar el trabajo auditivo en las aulas de una forma adecuada.

Si hacemos una reflexión acerca de cuál puede ser la causa o el motivo de que se hayan producido unas diferencias tan significativas entre el porcentaje de maestros y alumnos, en aspectos formativos básicos de la educación musical y en concreto de la percepción auditiva, y en el que los alumnos, en general, obtienen mejores porcentajes, cabe pensar que las acciones realizadas, derivadas de la primera fase de la investigación, han producido los resultados apetecidos.

Dichas acciones, que incluimos aquí con carácter de propuestas, se han orientado hacia la formación inicial y hacia la formación permanente.

En la formación inicial el primer paso ha de ser la revisión en profundidad de las guías docentes. Con la nueva estructura de los estudios de Grado y la consiguiente reducción del número de créditos dedicados a la Música en el Grado en Maestro de Educación Infantil, se hace indispensable un análisis de la práctica docente con el objeto de acercarla y adecuarla lo más posible a las necesidades expresadas por los encargados de llevar a cabo esta tarea en las aulas de Educación Infantil.

En los nuevos planes de estudio nos hemos visto obligados a reducir los contenidos de nuestra materia pero ello no debe ir en detrimento de la calidad de nuestra enseñanza sino que debe suponer una selección de aquellos contenidos considerados más relevantes y un cambio en el enfoque metodológico que conjugue teoría y aplicación práctica de la misma. En este sentido coincidimos plenamente con Bernal (1999: 25) cuando señala que: "Es necesario buscar una dimensión más operativa con el fin de que se propicie el aprendizaje efectivo [...]. La actuación educativa no debe ir en la línea de cuánto se sabe de música sino qué se sabe hacer con la música". Y continúa indicando que, aunque la cantidad de los conocimientos musicales que posea el especialista en Educación Infantil es muy importante, es más significativa la calidad de dichos conocimientos y la capacidad de adaptarlos para llevarlos a la práctica en el aula. Se trata entonces no de proporcionar a los alumnos numerosos contenidos musicales sino de dotarlos de recursos y métodos que los capaciten para trabajar la música, y más concretamente la percepción auditiva, en el aula infantil para poder aprovechar todas las posibilidades educativas que una y otra ofrecen.

Un segundo paso tendría que ver con la forma en que vamos a impartir nuestra docencia, es decir, con la metodología que vamos a emplear. Nuestra propuesta consiste en llevar a cabo una docencia que incida, sin olvidar la importancia de la teoría, en una formación más orientada a la práctica, que combine destrezas musicales y metodologías musicales específicas de la etapa junto con un trabajo didáctico encaminado a lograr una visión lo más práctica y realista posible sobre las diferentes formas de integrar el trabajo musical en el quehacer diario del trabajo en el aula. 
En la formación permanente los resultados y conclusiones de la primera fase de la investigación orientaron una serie de acciones, iniciadas ya el pasado curso 20122013, tales como: organización de cursos referidos a las diferentes metodologías y métodos de educación musical con aplicación en la etapa infantil, cursos para trabajar los distintos bloques sobre los que se asienta la educación musical de la etapa y la puesta en marcha de un Blog con el objetivo de facilitar el trabajo diario y favorecer la integración de la expresión musical en el aula de Educación Infantil.

Tal vez estos aspectos deban ser objeto de un estudio posterior más fundamentado que deba tenerse en cuenta en un futuro, abriéndose nuevas líneas de investigación que sin duda pueden reportar interesantes datos que contribuyan al enriquecimiento de la investigación que hasta ahora se lleva realizada.

Creemos firmemente que las Universidades deben revisar periódicamente sus planes de estudio para responder a las demandas de la sociedad en la que se insertan, por lo que el contacto entre ambos contextos debe ser permanente. Así mismo pensamos que de cara a asegurar la significatividad de los aprendizajes, la docencia universitaria, y concretamente en la materia que nos ocupa, ha de adecuarse a las necesidades reales del colectivo al que se dirige y a las necesidades relacionadas con la educación y la formación permanente.

Por último, queremos acabar con la siguiente reflexión:

Que la música es un importante elemento educacional desde los primeros años de vida del niño es un hecho constatado, sin embargo, en muchas ocasiones seguimos estando ajenos a esta realidad. El niño nace con una disposición natural hacia el hecho sonoro y musical, los investigadores así lo constatan en los cada vez más numerosos estudios realizados. Solo cabría entonces preguntarnos, ¿estamos enseñando en nuestras universidades acorde con los resultados de las investigaciones actuales? [...] (Díaz, 2004a: 118)

Como profesores que estamos formando a los futuros maestros, debemos esforzarnos por potenciar todas aquellas competencias del ámbito musical que tienen una clara repercusión en el desarrollo integral del niño, lo cual supone un gran reto. Es el momento de que la educación musical adquiera el protagonismo que se merece y no quede como algo residual en los actuales planes de estudios de Maestros, lo cual supone una contundente implicación por nuestra parte.

\section{Referencias bibliográficas}

AKOSCHKY, J. (1996): La audición sonora y musical en la Educación Infantil, en Eufonía, no4, Barcelona: Graó. p. 97-102.

BERNAL, J. (1999): La formación musical del maestro especialista en la Educación Infantil, en Eufonía, no 25, Barcelona: Graó. p. 23-32.

CALAFI RIUS, M. et all. (2004): Percepción auditiva del lenguaje. Programa para su entrenamiento. (5 elementos). Barcelona: Ars Médica. 
DÍAZ, M. (2004a): La educación musical en la etapa 0-6 años, itinerarios y proyecciones: Formación Inicial del Profesorado en la Etapa Infantil en España, en Resúmenes de la XXVI Conferencia Internacional ISME. Tenerife, p. 117-127.

LÓPEZ DE LA CALLE SAMPEDRO, M. A. (2008): La formación del maestro de educación infantil en la música y su enseñanza y su adecuación al espacio europeo de educación superior, en Innovación Educativa, $\mathrm{n}^{\circ}$ 18, Universidad de Santiago de Compostela: SPIC. p. 223- 237.

RUIZ, E. (2011): Expresión musical en Educación Infantil. Orientaciones didácticas. Madrid: CCS.

4.1. Articulos en publicaciones web

BERNAL, J. (2000): Implicaciones de la música en el currículum de educación infantil. Disponible en: http://musica.rediris.es/leeme/revista/bernal00.pdf. [0806- 2013]

DÍAZ, M. (2004b): La educación musical en la etapa 0-6 años", en Léeme, Lista Europea de Música en la Educación, $\mathrm{n}^{\circ} 14$. Disponible en: http://musica.rediris. es/leeme/revista/diaz04.pdf. [03- 06- 2013]

LÓPEZ DE LA CALLE SAMPEDRO, M. A. (2009): La formación de los maestros de Educación Infantil para la comprensión de la Música y su uso didáctico en Galicia. Disponible en: http://www.aufop.com/aufop/uploaded_files/articulos/1240872964.pdf. [08- 06- 2013]

\section{Los autores}

$\mathrm{M}^{\mathrm{a}}$ Esther Ruiz Palomo. Licenciada en Pedagogía y Doctora en Ciencias de la Educación, es además Profesora de E.G.B., maestra especialista en Educación Musical y Profesora de Piano. Posee una amplia experiencia docente en todos los niveles educativos. Actualmente es Profesora Ayudante Doctor en la Universidad de Burgos. Es autora de tres libros, varios capítulos de libros y distintos artículos y ha creado un blog de educación musical para educación infantil. Sus investigaciones giran en torno a la música como herramienta para prevenir y/o rehabilitar problemas de habla, la educación musical en la etapa infantil, la formación musical de los maestros de dicha etapa educativa y el poder socializador de la música.

Fernando Lara Ortega. Maestro, Doctor en Filosofía y Ciencias de la Educación. Especialidad: Filosofía: Lógica y Metodología de la Ciencia-Filosofía del Lenguaje. Catedrático de Escuela Universitaria de Psicología Evolutiva y de la Educación en la Universidad de Burgos. Veintinueve años de docencia e investigación universitaria en los temas propios de Psicología del Desarrollo y en Técnicas de investigación y Análisis de datos. Multitud de investigaciones y publicaciones en libros y revistas, en que se ha responsabilizado principalmente del diseño de la investigación, muestreo y análisis de datos. Dieciséis Tesis Doctorales dirigidas y otras en proceso de dirección. 
Rosa $\mathrm{M}^{\mathrm{a}}$ Santamaría Conde. Licenciada en Filosofía y Ciencias de la Educación y Doctora en Pedagogía. Durante once años ha ejercido como maestra funcionaria en los distintos niveles de educación obligatoria y en Equipos de Orientación Educativa y Psicopedagógica. Actualmente, es Profesora titular de la Universidad de Burgos en el Departamento de Ciencias de la Educación. Destacan distintas publicaciones de artículos en revistas especializadas, inclusive de algunos libros, el último de ellos Organización y planificación escolar de la editorial Síntesis. Sus últimas investigaciones han girado en torno a la enseñanza superior en el espacio europeo, la educación en valores y la organización y gestión de centros educativos. 